\title{
OTIMIZAÇÃO DA SOLDAGEM DE LIGAS DISSIMILARES DE AÇO INOXIDÁVEL 314 E COBRE CROMO C18200*
}

\author{
Mariana Tomé da Silva ${ }^{1}$ \\ Sheila Medeiros de Carvalho ${ }^{2}$ \\ Milton Sérgio Fernandes de Lima $^{3}$
}

\section{Resumo}

A câmara de empuxo do Motor Foguete a Propelente Líquido (MFPL) L75 é composta de uma liga Cobre cromo, classe C18200. A jaqueta de refrigeração, responsável pela extração de calor, é feita de aço inoxidável AISI 316L. No que tange ao processo de fabricação, a união entre a câmara de combustão e a jaqueta de refrigeração é uma etapa crítica, pois envolve processos de união de materiais dissimilares e de componentes de geometria complexas. Este trabalho visa contribuir à fabricação do conjunto motor foguete, por meio da soldagem a laser entre a câmara e a jaqueta. Com o uso de um laser a fibra de alta potência, foi possível verificar que existe uma condição otimizada de solda sobreposta, inox sobre $\mathrm{Cu}(\mathrm{Cr})$, com potências entre 1.000 e $1.300 \mathrm{~W}$ e velocidades de soldagem de $0,05 \mathrm{~m} / \mathrm{min}$ a $1 \mathrm{~m} / \mathrm{min}$. Embora as análises mostrem heterogeneidade microestrutural, pela dissolução parcial de uma fase na outra, obteve-se níveis de dureza aproximadamente constantes ao longo da profundidade da junta.

Palavras-chave: Soldagem a laser; Ligas de cobre; Aços inoxidáveis; Soldagem dissimilar.

\section{WELDING OPTIMIZATION OF DISSIMILAR ALLOYS 314 STAINLESS STEEL ANDCOPPER CHROMIUM C-18200}

\section{Abstract}

The thrust chamber of the L75 liquid propelled rocket engine is composed of a copper chrome alloy, C18200 class. The cooling jacket, responsible for heat extraction, is made of AISI 316L stainless steel. With respect to the manufacturing process, the union between the combustion chamber and the cooling jacket is a critical step because it involves joining of dissimilar materials and complex geometry components. This work aims to contribute to the manufacture of the motor assembly, by means of laser welding between the camera and the jacket. With the use of a high power laser fibre was possible to verify that there is an optimal condition of overlay welds of stainless steel on $\mathrm{Cu}(\mathrm{Cr})$ usinglaser power from 1,000 to $1,300 \mathrm{~W}$ and welding speed between $0.5 \mathrm{~m} / \mathrm{min}$ to $1 \mathrm{~m} / \mathrm{min}$. Although it had been observed microstructural heterogeneity, because of the partial dissolution of one phase in the other,was obtained almost constant hardness levels along the depth of the joint.

Keywords: Laser beam welding; Copper alloys; Stainless steels; Dissimilar welding.

1 Aluna de graduação/Engenharia de Materiais, Universidade Federal de São Paulo, Campus São José dos Campos, São Paulo, Brasil.

2 PhD/Engenharia Metalúrgica, Tenente-engenheira, Laboratório de Propulsão Espacial/Instituto de Aeronáutica e Espaço, Departamento de Ciência e Tecnologia Aeroespacial, São José dos Campos, São Paulo, Brasil.

3 PhD/Físico, Chefe da Sub-divisão de Lasers, Divisão de Fotônica/Instituto de Estudos Avançados, Departamento de Ciência e Tecnologia Aeroespacial, São José dos Campos, São Paulo, Brasil. 


\section{INTRODUÇÃO}

O desenvolvimento do Motor Foguete a Propelente Líquido (MFPL) L75 é baseado em modernos e complexos processos tecnológicos, além de requerer investimentos em longo prazo [1]. Nesse contexto, a especificação, o projeto e a construção do MFPL L75 é fruto de uma parceria entre o Instituto de Aeronáutica e Espaço (IAE), a Agência Espacial Brasileira (AEB), e a Agência Espacial Alemã (DeutschesZentrumfür Luft-undRaumfahrt - DLR), através do convênio SICONV $\mathrm{n}^{\circ}$ $755573 / 2012$.

Os principais componentes do motor MFPL L75 são o sistema de alimentação, o sistema de combustão, o sistema de ancoragem, o sistema de ignição e o sistema de controle. No que tange aos processos de fabricação, a câmara de combustão é de particular interesse. Nela ocorre a queima do propelente, produzindo gases que podem atingir temperaturas da ordem de $3.300{ }^{\circ} \mathrm{C}$. Dessa forma, no processo de fabricação dessa câmara, há a necessidade de emprego de materiais com elevada condutibilidade térmica, bem como elevada resistência mecânica em tração em altas temperaturas $\left(300^{\circ} \mathrm{C}\right)$.

O material utilizado na câmara de combustão do MFPL L75 é o cobre cromo C18200, por apresentar alta condutividade térmica e elevada resistência mecânica em tração quando tratado termicamente [2]. Visando aumentar a taxa de extração de calor da câmara de combustão, esta é envolta por uma jaqueta de refrigeração, fabricada de aço inoxidável AISI 316L [3-5]. O AISI $316 \mathrm{~L}$ possui um ótimo desempenho, tanto em altas temperaturas, quanto em baixíssimas temperaturas, bem como excelente resistência a corrosão, ductilidade e soldabilidade [6].

O aço AISI 316L e a liga Cobre-Cromo 18200 também foram utilizados na câmara de empuxo do motor foguete a propelente líquido $L 15$, uma versão anterior a do MFPL L75. Na ocasião, utilizou-se o processo de soldagem a arco elétrico com eletrodo não consumível de Tungstênio, também conhecido como processo de soldagem TIG (Tungsten Inert Gas). Após a soldagem dos componentes foi observado que houve aquecimento de uma região de cerca de 40 milímetros além da zona fundida (ZF), a qual media $3 \mathrm{~mm}$. Os ensaios em tração demonstraram que o conjunto soldado apresentou resistência a tração de $60 \%$ do metal base (CobreCromo C18200). Baseado nesses resultados, o processo de soldagem TIG foi descartado.

Como o aporte térmico na soldagem TIG é elevado, quando comparado com os processos de alta densidade de energia, o cordão de solda é largo e mais suscetível a defeitos de soldagem $[7,8]$. Existe também a possibilidade do surgimento de fases frágeis, uma vez que a liga de Cobre-Cromo C18200 apresenta precipitados dispersos que são instáveis em altas temperaturas [9-11].

A extensão da região fragilizada varia com o processo de soldagem, isto é, quanto menor o aporte térmico, menor é a zona termicamente afetada e, consequentemente, menor tendência à falha por trincamento [12,13].

Para resolver estes problemas, é necessário optar por um processo de soldagem que minimize a extensão da zona termicamente afetada (ZTA). Nesse aspecto, os melhores resultados são obtidos por soldagem por feixe de elétrons (ElectronBeamWelding - EBW) e pela soldagem a laser (Laser BeamWelding - LBW) $[13,14]$. O método EBW não será considerado, pois necessita de vácuo durante a soldagem, o que é impeditivo para o componente em questão.

A soldagem a laser tem sido estudada no Departamento de Ciência e Tecnologia Aeroespacial, mais especificamente no Instituto de Estudos Avançados (IEAv). O 
IEAv acumula mais de dez anos de experiência na soldagem de componentes estruturais aeroespaciais, inclusive ligas de ultra-alta resistência, ligas de titânio e materiais dissimilares [15-17].

Mai e Spowage [18] investigaram a soldagem a laser entre o aço AISI 304 e o Cobre, utilizando um laser de Nd:YAG de $350 \mathrm{~W}$ de potência. Os melhores resultados foram obtidos com a focalização do laser no aço inoxidável, resultando em menos de $2 \%$ de cobre diluído na zona fundida do aço inox, ausência de microtrincas nas zonas fundida e termicamente afetada, além de uma microestrutura dendrítica na zona fundida com a presença de martensita.

Igualmente, Tosto e colaboradores [19] também soldaram o aço AISI 304 L com o cobre, porém o processo utilizado foi o por feixe de elétrons. As juntas de topo possuíam $25 \mathrm{~mm}$ de espessura. Esses autores também obtiveram um cordão de solda livre de porosidades e microtrincas, tanto na ZF quanto na ZTA, porém eles enfatizaram que podem surgir problemas em serviço devido à instabilidade da microestrutura observada, nas temperaturas de operação do conjunto.

Magnabosco e colaboradores [20] obtiveram juntas soldadas do aço inox AISI 304L e 316 L com a liga de cobre (UNS C 11000), em diferentes espessuras, unidas por feixe de elétrons. A junta realizada em um único passe não apresentou defeitos de soldagem. Porém, naquelas juntas realizadas em dois passes, observaram-se porosidades na fase rica em cobre, além de microtrincas intergranulares na interface entre a ZF e o aço inoxidável. Os autores relataram que a otimização dos parâmetros de soldagem pode minimizar a ocorrência desses defeitos.

Espera-se que a tecnologia de soldagem a laser possa ser aplicada na manufatura dos componentes da câmara de combustão, de forma a substituir com vantagens o processo TIG manual.

\section{MATERIAIS E MÉTODOS}

Os materiais utilizados no presente estudo foram chapas de aço inox 304, de $0,75 \mathrm{~mm}$ de espessura e chapas de cobre cromo C18200, de 2,18 $\mathrm{mm}$ de espessura. A chapa de cobre cromo C18220 foi fornecida pela empresa Artisa Metais EIRELI EPP, na forma de placas $10 \mathrm{~mm}$ de espessura e em seguida usinada na divisão de mecânica do IAE, até a espessura final de $2,18 \mathrm{~mm}$. Os materiais base apresentaram dureza Vickers de $130 \mathrm{HV}$, para o cobre cromo e de $230 \mathrm{HV}$, para o inox 304.A composição nominal do aço inox AISI 304 e do cobre cromo C1820 são apresentadas nas Tabelas 1 e 2 , respectivamente.

Tabela 1. Composição da norma do aço 304 (\%p).

\begin{tabular}{cccccccc}
\hline $\mathbf{C}$ & $\mathbf{M n}$ & $\mathbf{S i}$ & $\mathbf{P}$ & $\mathbf{S}$ & $\mathbf{C r}$ & $\mathbf{N i}$ & $\mathbf{N}$ \\
\hline 0,07 & 2,00 & 0,75 & 0,045 & 0,015 & $17,5 \mathrm{~A} 19,5$ & $8,0 \mathrm{~A} 10,5$ & 0,10 \\
\hline
\end{tabular}

Tabela 2. Composição nominal do cobre cromo C18200 (\%p).

\begin{tabular}{ccccccccccc}
\hline $\mathbf{C u}$ & $\mathbf{N i}$ & $\mathbf{P b}$ & $\mathbf{S n}$ & $\mathbf{P}$ & $\mathbf{A l}$ & $\mathbf{F e}$ & $\mathbf{Z n}$ & $\mathbf{Z r}$ & $\mathbf{C r}$ & $\mathbf{S i}$ \\
\hline 99,139 & 0,005 & 0,005 & 0,005 & 0,007 & 0,008 & 0,02 & 0,032 & 0,072 & 0,704 & 0,003 \\
\hline
\end{tabular}

A seguir, segue uma breve descrição dos métodos empregados nesse trabalho.Todas as análises e processos envolvidos no presente estudo foram realizados nos laboratórios da Divisão de Fotônica (EFO) do Instituto de Estudos Avançados (IEAv). 


\subsection{Soldagem}

O laser a fibra utilizado neste trabalho está instalado no Laboratório Multiusuário de Desenvolvimento de Aplicações de Laser e Ótica (DEDALO) da Divisão de Fotônica (EFO) do Instituto de Estudos Avançados (IEAv). Trata-se de um laser de $2 \mathrm{~kW}$ de potência média (IPG, Modelo YLR-2000) com comprimento de onda de 1,07 $\mu \mathrm{m}$. Este laser é dotado de uma fibra de saída com $50 \mu \mathrm{m}$ de diâmetro e $5 \mathrm{~m}$ de extensão. Uma unidade de acoplamento de fibras faz a conexão de uma segunda fibra com $100 \mu \mathrm{m}$ de diâmetro e $10 \mathrm{~m}$ de comprimento que é utilizada nos processos de soldagem. A fibra secundária é conectada a um colimador óptico formando o sistema de acoplamento do feixe.

Os experimentos de soldagem foram realizados em uma mesa com comando numérico computadorizada (CNC), por meio do software EMC2 e acionada por motores de passo, com velocidade máxima de $160 \mathrm{~mm} / \mathrm{s}$ e cursos dos eixos $X$ de $430 \mathrm{~mm}$ e $Y$ de $508 \mathrm{~mm}$, ambos com resolução de $5 \mu \mathrm{m}$. Um eixo $Z$ com curso de $215 \mathrm{~mm}$ e resolução de $1 \mu \mathrm{m}$ foi incorporado à mesa. Este eixo fixa a óptica de focalização e facilita posicionar corretamente o foco do feixe de laser sobre a amostra. No caso das chapas de $5 \mathrm{~mm}$ de espessura, o foco do laser foi posicionado a superfície da chapa superior.

Antes da realização do processo de soldagem, as chapas de inox e de cobre cromo foram lixadas com a lixa de granulometria $600 \mu \mathrm{m}$. O tipo de junta utilizado na soldagem foi sobreposta. A liga de aço inox foi sobreposta $10 \mathrm{~mm}$ sobre a liga de cobre cromo C18200, conforme Figura 1. Sendo assim, o feixe de laser incidiu diretamente sobre a chapa de inox 304.

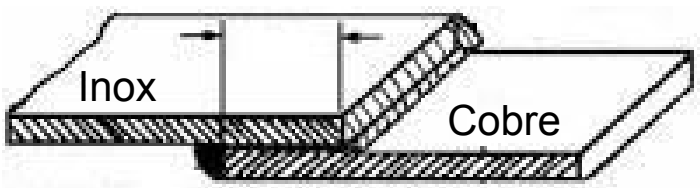

Figura 1. Desenho representativo de junta sobreposta.

A fim dese verificar qual era a melhor condição de soldagem, foi realizada uma carta de processo de soldagem. A carta de processo associou dois parâmetros de soldagem (velocidade e potência), a uma determinada qualidade da solda (parâmetros de saída). Os parâmetros de saída foram a penetração e o aspecto superficial. A Tabela 3 apresenta os parâmetros de processos utilizados.

Tabela 3. Carta de processo de soldagem.

\begin{tabular}{ccc}
\hline $\begin{array}{c}\text { Identificação dos } \\
\text { corpos de prova }\end{array}$ & $\begin{array}{c}\text { Potência } \\
\text { (W) }\end{array}$ & $\begin{array}{c}\text { Velocidade de soldagem } \\
\text { (m/min) }\end{array}$ \\
\hline CDP_1000W_1m_min & 1000 & 1 \\
\hline CDP_1200W_1m_min & 1200 & 1 \\
\hline CDP_1300W_1m_min & 1300 & 1 \\
\hline CDP 1400W_1m_min & 1400 & 1 \\
\hline CDP_1200W_1,5m_min & 1200 & 0,5 \\
\hline CDP_1200W_0,5m_min & 1200 & \\
\hline
\end{tabular}




\subsection{Embutimento}

O embutimento é utilizado para proteger materiais frágeis e para produzir amostras de tamanho uniforme. A chapa soldada na etapa 2.1 foi dividida em 3 corpos de prova, sendo embutida, dessa forma, duas soldas por vez. Foi utilizada a resina fenólica baquelite. O processo de embutimento foi a quente e a vácuo. Após as peças serem embutidas, as amostras foram identificadas de acordo com a Tabela 3.

\subsection{Lixamento}

As amostras foram lixadas em água corrente. As lixas utilizadas eram da marca 3M. A granulometria utilizada seguiu a sequência degrau 220 a 1200.

O processo foi realizado com a fixação da lixa em uma politriz da marca AROTEC, modelo AROPOL $2 \mathrm{~V}$. O sentido do lixamento das peças foi alternado de $90^{\circ}$, visando assim retirar os ricos deixados pela lixa anterior. Foi utilizado um microscópio OLYMPUS, modelo TH-4 200, para verificar se havia nas amostras, riscos remanescentes do lixamento anterior.

\subsection{Polimento}

O polimento ocorre após a etapa de lixamento das peças, sendo realizado também na politriz AROTEC, modelo AROPOL $2 \mathrm{~V}$. Diferentemente do lixamento, no polimento o corpo de prova é submetido a movimentos circulares. As peças foram polidas com o pano especial de polimento e pasta de diamante de $6 \mu ; 3 \mu ; 0.5 \mu$ e $0.25 \mu$, da marca AROTEC. A cada mudança da espessura da pasta, o pano de polimento foi trocado e a peça analisada no microscópio.

\subsection{Ataque químico}

Após o término do polimento, as peças foram uma a uma atacada quimicamente. A solução de ataque utilizada foi a de cloreto férreo (5 partes de $\mathrm{FeCl}_{3} ; 50$ partes de HCL; 100 partes de água destilada). O ataque ocorreu por fricção da solução de ataque sob a superfície do corpo de prova durante o tempo de 10 segundos. Em seguida, a amostra foi lavada com água corrente, álcool etílico, e seca com jatos de ar comprimido.

\subsection{Metalografia}

As análises foram realizadas em um microscópio ZEISS. Neste equipamento foram obtidas as imagens das peças atacadas pelo cloreto férrico. A magnificação das imagens variou de $25 \mathrm{X}$ a $500 \mathrm{X}$.

\subsection{Microdureza}

A microdureza Vickers baseia se na resistência que o material oferece à penetração de uma pirâmide de diamante de base quadrada e ângulo entre faces de $136^{\circ}$, sob uma determinada carga. O valor de microdureza Vickers (HV) é o quociente da carga aplicada $(F)$ pela área de impressão $(A)$ deixada no corpo ensaiado (Equação 1). 


$$
H V=F / A
$$

Nesse processo, foi analisada a dureza por toda a extensão do cordão de solda ( zona fundida - ZF, zona termicamente afetada -zta e do metal base -MB). A carga utilizada foi $50 \mathrm{gf}$ e o tempo de endentação foi de 9s. A dureza foi medida primeiramente por toda a extensão da solda para ser analisada a influência do processo de soldagem em cada material. A distância de uma medida para outra foi de $0.001 \mathrm{~mm}$, a força aplicada de $50 \mathrm{~g} /$ força e o tempo de 9 segundos.

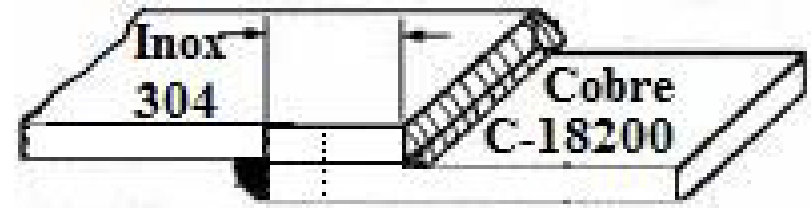

Figura 2 - Sentido da medição da Microdureza Vickers nos cordões de solda.

\section{RESULTADOS E DISCUSSÃO}

A Figura 3 apresenta as micrografias das seções transversal das soldas. Verifica-se que a condição CDP_1000W_1m_min não produz união metalúrgica entre o aço e o cobre. Na região de união Figura $3 b$ é observado uma pequena união, com poro central. Na condição CDP_1200W_1m_min (Figura 3d) a uniãofaz-se mais visível e as zonas ricas em $\mathrm{Fe}$ e Cu estão visíveis na Figura 3e. A partir desta condição, tanto diminuindo a velocidade de soldagem para $0,5 \mathrm{~m} / \mathrm{min}$ (Figuras $3 \mathrm{~g}$, 3h e $3 \mathrm{i}$ ) quanto aumentando-se a potência para 1300 W (Figuras 3j, 3k e 3l) é obtido boa mistura de fases.

Não foram observadas trincas ou porosidades na extensão da seção transversal das soldas apresentadas na Figura 3.

O gráfico da Figura 4 correlaciona a potência de soldagem com a penetração, para as condições CDP_1000W_1m_min, CDP_1200W_1m_min e CDP_1300W_1m_min. Analisando os dados apresentados na Figura 3, observa-se que à medida que ocorre o aumento da potência do laser, há o aumento da penetração até o limite de espessura das chapas. 


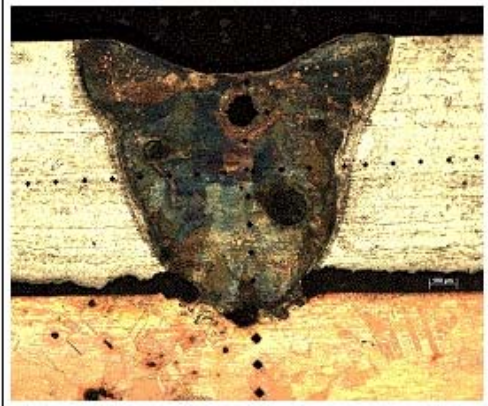

(a)

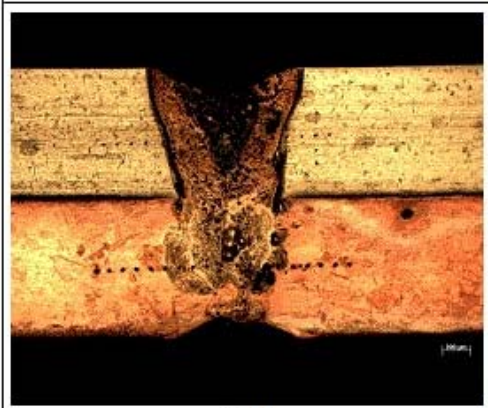

(d)

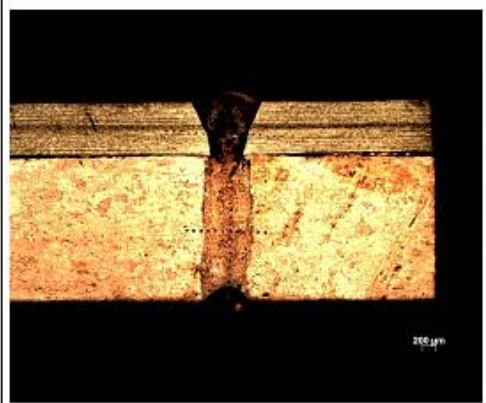

(g)

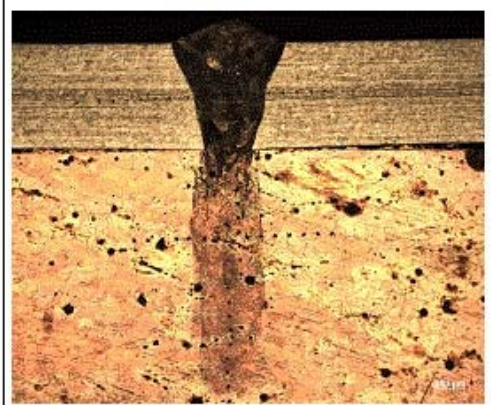

(j)

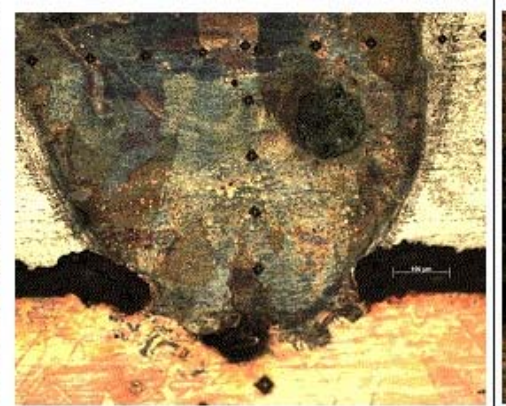

(b)

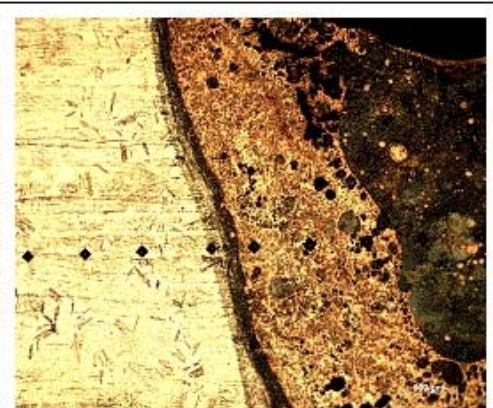

(e)

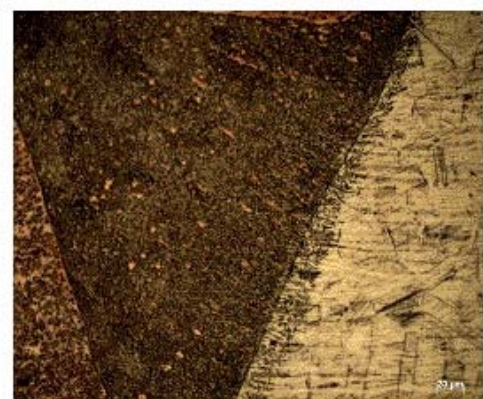

(h)

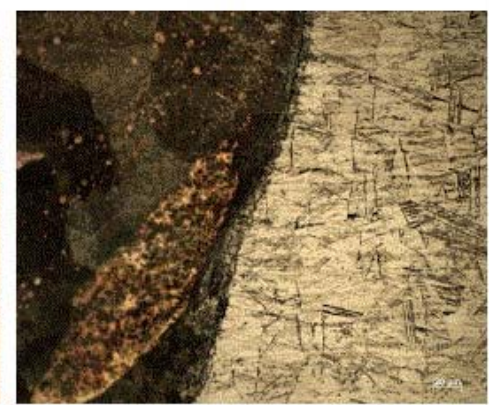

(k)

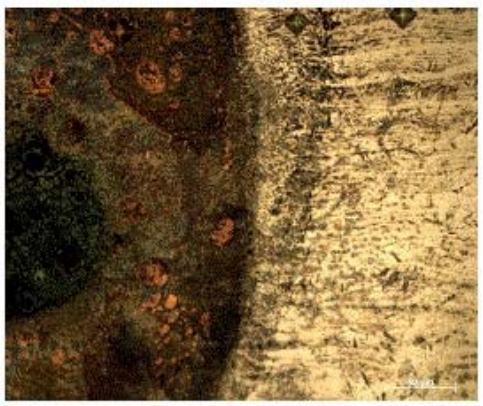

(c)

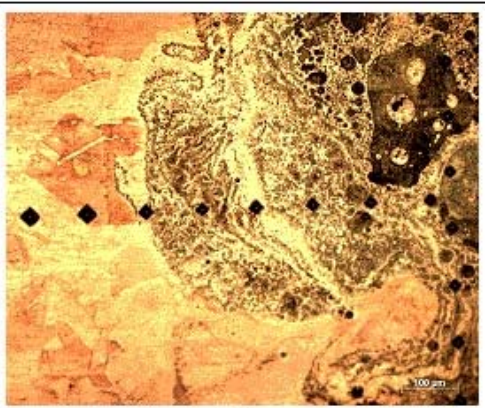

(f)

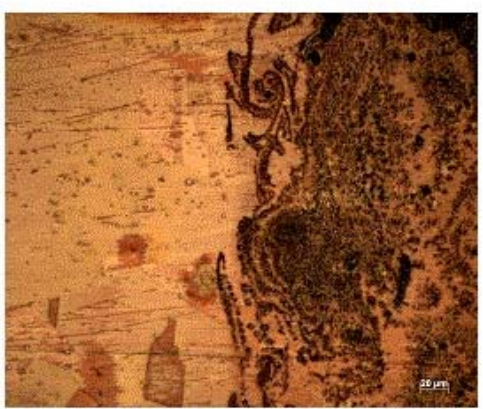

(i)

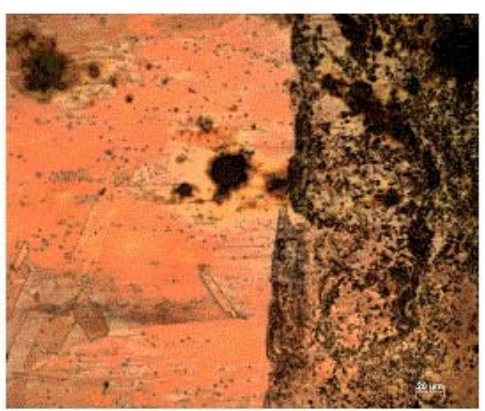

(I)

Figura 3. Micrografias das soldas dissimilares de inox $304 \mathrm{com}$ cobre cromo $\mathrm{C} 18200$. a)Cordão de solda CDP_1000W_1m_min; b) Centro da solda CDP_1000W_1m_min; c) Interface da zona fundida e metal base Inox da CDP 1000W_1.0m_min; d)Cordão de solda CDP_1200W_1m_min; e) Interface da zona fundida e metal base Inox da CDP $1200 \mathrm{~W} 1 \mathrm{~m}$ min; f) Interface da zona fundida e metal base CCr-18200 da CDP 1000W 1.0m min; g)Cordão de solda CDP $1200 \mathrm{~W}$ 0.5m min; h) Interface da zona fundida e metal base Inox da CDP_1200W_0.5m_min; i) Interface da zona fundida e metal base CCr-18200 da CDP_1200W_0.5m_min; j)Cordão de solda CDP_1300W_1m_min; k) Interface da zona fundida e metal base Inox da CDP_1300W_1m_min; I) Interface da zona fundida e metal base CCr-18200 da CDP_1300W_1.0m_min. 


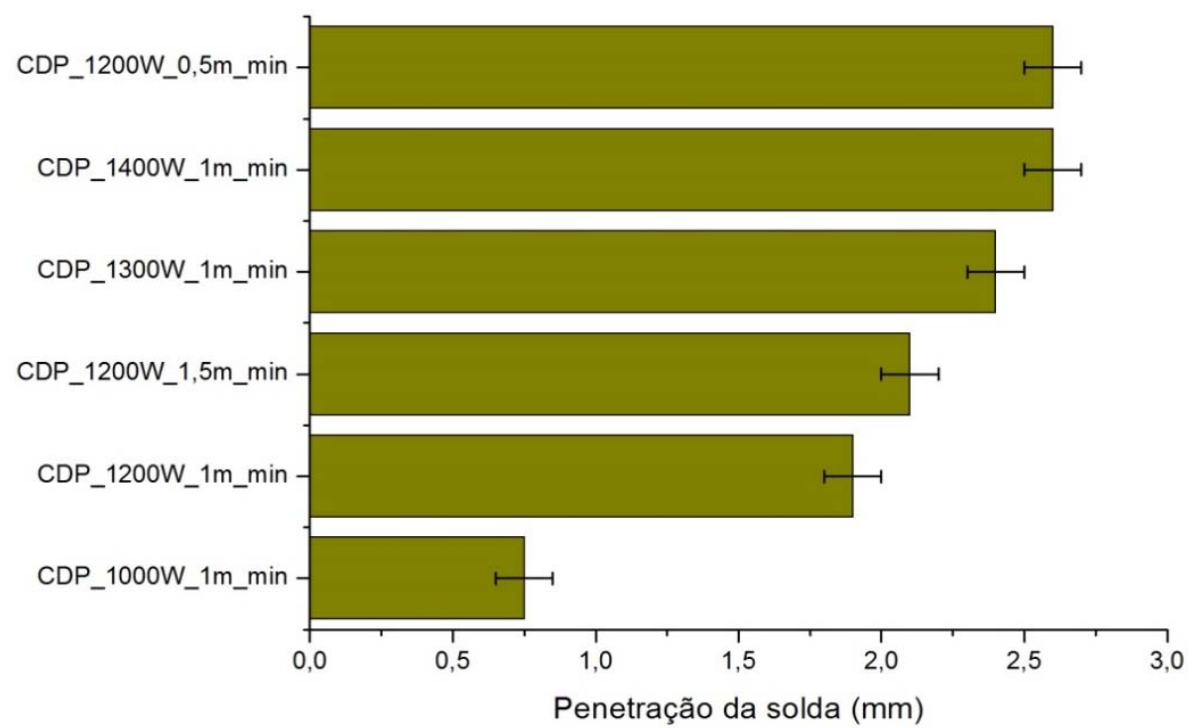

Figura 4. Gráfico correlacionando condição de soldagem e penetração.

A Figura 5 apresenta os valores de dureza Vickers ao longo do sentido de penetração. Observa-se na Figura 5 que a dureza diminui ao longo da penetração, uma vez que as medidas se iniciam na chapa de inox e terminam na chapa de cobre cromo C18200. A dureza na solda na região do inox é menor do que a encontrada no inox longe da fusão (metal base). $O$ cobre tem efeito austenitizante na estrutura do ferro, portanto, há a tendência de se observar austenita na região solidificada do inox, com muito pouca ou nenhuma ferrita. Isto explica os valores baixos de dureza na zona fundida do inox.

- CDP_1000W_1m_min $\rightarrow$ CDP_1200W_1m_min $\rightarrow$ CDP_1200W_0.5m_min $\rightarrow$ CDP_1300W_1m_min

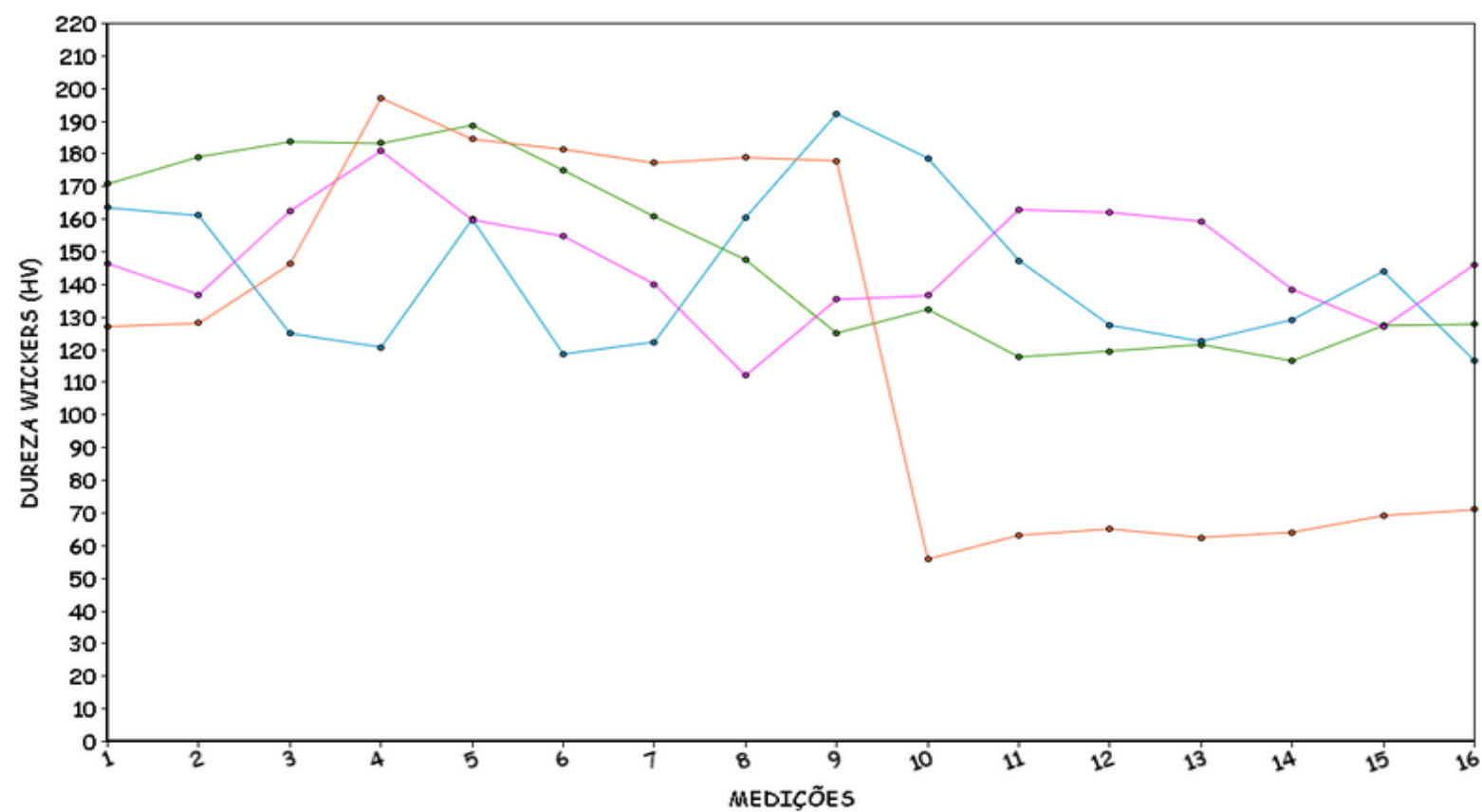

Figura 5 -Microdureza Vickers referente aos cordões de solda

Nota-se na Figura 5 que, para condição CDP_1000W_1m_min, o perfil de dureza apresenta uma queda brusca após a região de transição do inox com cobre cromo. 
Isso ocorre, uma vez que nessa condição a energia de soldagem foi insuficiente para fundir completamente as duas chapas.

A dureza apresentada na região cobre cromo C18200 é cerca de $60 \mathrm{HV}$. No entanto, esse valor é abaixo do valor encontrado para a dureza do metal base do cobre cromo. Provavelmente, houve calor suficiente para solubilizar a fase cromo nos grãos, mas sem tempo suficiente para precipitação no estado sólido. Portanto, o amolecimento pode ter acontecido por perda de precipitados de cromo.

Nas condições CDP_1200W_1m_min, CDP_1200W_0,5_m_min e CDP_1300W_1m_min, observa-se uma oscilação nas médidas de dureza ao longo do perfil. Essa oscilação ocorre devido à dissolução incompleta das fases na zona fundida. Como se vê na Figura 3, as zonas fundidas apresentam glóbulos de cobre na linha doinox.

\section{CONCLUSÃO}

Após a realização das análises de soldagem entre o aço inoxidável 316 L e liga cobre cromo, é possível determinar que:

As melhores condições de soldagem, considerando qualidade metalúrgica, formato dos cordões e penetração total é aquela com potência de $1200 \mathrm{~W}$ e $0,5 \mathrm{~m} / \mathrm{min}$ ou $1300 \mathrm{~W}$ e $1 \mathrm{~m} / \mathrm{min}$.

O perfil de dureza obtido nas condições otimizadasmostrou-se uniforme em profundidade, o que pode ser um indicativo de boas propriedades mecânicas da junta.

\section{Agradecimentos}

A autora MTS agradece a bolsa de iniciação científica do programa PIBIC/CNPq do IAE.

\section{REFERÊNCIAS}

1 Almeida, J. da E. "Especificação do sistema de partida da turbina da turbobomba do Motor Foguete a Propelente Líquido L75”, IAE, São José dos Campos, 2011. (Relatório Técnico 003-430000-B2001).

2 Santana JR. A. Investigation of Passive ControlDevicestoSuppressAcousticlnstability in CombustionChambers. 2008. 165f. Thesis (Doctor in scienceofAeronauticsandMechanicsEngineering). Instituto Tecnológico de Aeronáutica, São José dos Campos.

3 Almeida, J. da E. Motor Foguete a Propelente Líquido L75. Seminário de Projetos de Pesquisa e Desenvolvimento, São José dos Campos, 2012.

4 Aerospace Technology and Management, Vol.1, No.1, pp.99-106. doi: 10.5028/jatm.2009.010199106.

5 GAHUN, G. G., 1989, "Constructionand design ofLiquidRocketEngines”, (in Russian), Moscow, Mashinostroenie Press, 424p.

6 Ribeiro, Rosinei Batista. Análise morfológica de pites em aços inoxidáveis austeníticos ABNT 304 e 310 submetidos à névoa salina. 249 f. Tese (Doutorado) - Curso de Engenharia Mecânica na área de Projetos e Materiais, Universidade Estadual Paulista, Guaratinguetá, 2004.

7 Ion, J. C. Laser Processing of Engineering Materials. Oxford: Elsevier, 2005. 556 p. ISBN 0750660791.

8 Marques P. V. et al. Soldagem: fundamentos e tecnologia. Belo Horizonte: UFMG, 2005. 363 p. ISBN: 85-7041-437-4. 
9 AWS Handbook. Metals and Their Weldability. 7th ed. Miami: American Welding Society, 1982. v. 4, p. 434 - 472, ISBN 0-87171-218-0

10 ASM International Handbook. Properties and selection: nonferrous alloys and specialpurpose materials, Metals Park: ASM International, 1990. v. 2

11 Lancaster, J.F. Metallurgy of welding. Cambridge: Abington Publishing, $1999.446 \mathrm{p}$.

12 Lima, M. S. F. Lasers in material processing. In: Lasers in Chemistry: Influencing Matter. Weinheim:Edited by Maximilian Lackner, 2008. p.1195 - 1209, ISBN: 978-3-527-319978.

13 Steen, W. M. Laser material processing. 2nd ed. London: Springer. 2001. p.346. ISBN: 3-540-76174-8

14 Scotti, A.; REIS, R. P. Fundamentos da prática da soldagem a plasma. São Paulo: ABS, 2007, 152 p.

15 Carvalho, S. M. ; Lima, M. S. F.. Laser Beam Welding Tempered 300M Ultrahigh Mechanical Strength Steel. Journal of the Brazilian Society of Mechanical Sciences and Engineering (Impresso) JcR, v. 34, p. 18-23, 2012.

16 Carvalho, S. M. ; Baptista, C. A. R. P.; Lima, M. S. F. . Simulating the damage accumulation in aircraft bleed system ducts joined by laser and arc welding processes.Procedia Engineering,.Procedia Engineering, v. v. 10, p. 1322-1327, 2011.

17 Siqueira, G. Soldagem a laser autógena da liga de alumínio aeronáutico AA6013: Otimização de parâmetros e Análise microestrutural. 2007. 105f. Dissertação (Mestrado) - Instituto Tecnológico de Aeronáutica, São José dos Campos, 2007.

18 Mai, T. A. and Spowage, A. C. Characterisation of Dissimilar Joints in Laser Welding of Steel-Kovar, Copper-Steel and Copper-Aluminium. Mater.Sci. Eng., A, 2004, 374: 224233.

19 Tosto, S., Nenci, F., Hu Jiandong, G., Corniani, F., Pierdominici. Microstructure of copper-AISI type 304L electron beam welded alloy. Mater.SciTechnol, 19, 2003, 519522.

20 Magnabosco I., Ferro P., Bonollo F., Arnberg L. An investigation of fusion zone microstructures in electron beam welding of copper-stainless steel. Materials Science and Engineering A, Volume 424, Issues 1-2, 25 May 2006, Pages 163-173 